\title{
Socio-Demographic and Clinical Profile of Health Care Workers Diagnosed for COVID-19 by Truenat at a Tertiary Care COVID Hospital
}

\author{
Bineeta Kashyap $^{1 * ®}$, Rajat Jhamb ${ }^{2}$, Narendra Pal Singh ${ }^{1}$, Krishna Sarkar' ${ }^{1}$ Rajnish Avasthi ${ }^{2}$, Ashwani \\ Khanna $^{3}$
}

\author{
${ }^{1}$ Department of Microbiology, University College of Medical Sciences and Guru Teg Bahadur Hospital, New \\ Delhi-110095, India \\ ${ }^{2}$ Department of Medicine, University College of Medical Sciences and Guru Teg Bahadur Hospital, New \\ Delhi-110095, India \\ ${ }^{3}$ State TB Officer, RNTCP, New Delhi-110011, India
}

*Corresponding Author: Bineeta Kashyap, M.D., Associate Professor, Department of Microbiology, University College of Medical Sciences and Guru Teg Bahadur Hospital, New Delhi-110095, India. Tel: +91-9899583514, Email: dr_ bineetakashyap@yahoo.co.in

Received November 12, 2020; Accepted December 25, 2020; Online Published January 8, 2021

\begin{abstract}
Background: In December 2019, in Wuhan, China; a new coronavirus emerged that had not been previously identified in humans. Hence is crucial to characterize the infection risk among infected health care workers (HCWs), being responsible for secondary transmission to patients, and others.

Objectives: The current study aimed to assess the disease burden among the front-line warriors and efficiently planned the preventive and management strategies for such infections.

Methods: HCWs with clinical suspicion of COVID-19 infection, who reported to Fever Clinic for possible diagnosis by Truenat testing, were enrolled through a self-reporting Risk Assessment form. An oropharyngeal swab was subjected to Truenat testing based on the principle of Real time reverse transcription polymerase chain reaction (RT-PCR).

Results: Doctors comprised $60 \%$ of our HCWs. Eighty-three percent of the HCWs under study reported either the presence of BCG scar or gave a history of BCG immunization at birth. The maximum number of HCWs $(29.16 \%)$ took Hydroxychloroquine prophylaxis for four weeks. Seventy-four percent of the HCWs affirmed the use of personal protective equipment (PPE) at the time of exposure. The most common mode of infection reported was the exposure to COVID-19 patients. Fever was the most common reported symptom. Truenat was positive in 9 of $100 \mathrm{HCWs}$ who were tested, giving an infection rate of $9 \%$.

Conclusion: The study provides insights into the burden of COVID-19 infection among HCWs, and guides us to evaluate and plan our preventive measures and management strategies for such infections.

Keywords: COVID-19, Health Care Workers, Reverse Transcription-Polymerase Chain Reaction, BCG, Hydroxychloroquine
\end{abstract}

\section{Background}

Human coronavirus can cause mild diseases such as common cold, while other severe respiratory viral diseases caused by them include Middle East respiratory syndrome (MERS) and severe acute respiratory syndrome (SARS). In December 2019, in the city of Wuhan, China; a new coronavirus emerged that had not been previously identified in humans. ${ }^{1}$ Fever, cough, breathlessness, and other respiratory complaints were the usual signs and symptoms, though, more severe cases showed pneumonia, severe acute respiratory syndrome, and occasionally death. The primary route for the spread of COVID-19 is through aerosol droplets that are expelled during coughing, sneezing, or breathing. However, concerns about possible airborne transmission also exist. ${ }^{2}$
Health care workers (HCWs) are exposed to hazards that put them at risk of infection with an outbreak pathogen as they are at the front line of any outbreak response. As doctors, health professionals, and other staff working in a health care institution are at the vanguard in the fight against COVID-19; it is of utmost importance that adequate measures are taken to ensure their safety. A study published on 21 May in JAMA Network Open, an open-access medical journal, showed that in China's Wuhan, the initial epicentre of the coronavirus outbreak, the incidence rate among healthcare workers was $1.1 \%$. As the fight against COVID-19 transmission in India has entered a critical phase, many doctors, nurses, ward staff, and other healthcare professionals have tested positive. By the steadily increasing number of patients, the medical

Copyright (C 2021 The Author(s). This is an open-access article distributed under the terms of the Creative Commons Attribution License (http:// creativecommons.org/licenses/by/4.0), which permits unrestricted use, distribution, and reproduction in any medium, provided the original work is properly cited. 
infrastructure is likely to be stretched. ${ }^{2}$ Foreign regional estimates also show that frontline HCWs may account for $10 \%-20 \%$ of all diagnoses. ${ }^{3}$ Such literature suggests the possibility for HCWs to perpetuate community transmission, even when they are asymptomatic or mildly symptomatic; and this justifies increasing the screening of HCWs. In a scenario when cases are rapidly shifting towards community transmission, it is very tedious to trace down or predict where the cases are originating from. Therefore, all measures should be implemented to ensure the lesser infectivity of healthcare workers. With ongoing transmission from both symptomatic and asymptomatic individuals, the burden of COVID-19 is expected to rise in the coming future. Consequently, there will be an ongoing need for dedicating the remaining healthcare workers and staff to more patient-facing roles. Because this requires close personal exposure to patients already infected with the virus, frontline HCWs will be, therefore, more susceptible to the infection, which may also contribute to further hospital-based transmission. ${ }^{4}$

\section{Objectives}

Despite increasing reports of COVID-19 among HCWs, there is a paucity of published research articles, either prospective or retrospective. To the best of our knowledge, no study has been published on the utility of Truenat testing for COVID-19 among HCWs. Hence, this study was initiated to study the positivity for COVID-19 infection among HCWs at a tertiary care COVID-19 hospital and to correlate the demographic, social, and clinical characteristics of the HCWs with their positivity for COVID-19. This would provide us with insights into the burden of COVID-19 infection among HCWs, and guides us to evaluate and further plan our preventive measures and management strategies for such infections.

\section{Methods}

The study was conducted in an Advanced Virology laboratory and COVID-19 testing Centre at University College of Medical Sciences (UCMS) \& GTB hospital $(\mathrm{GTBH})$, a tertiary care hospital in the east part of Delhi, India; from 7th July to 15th August. The hospital is a dedicated COVID-19 hospital.

The participants were all the HCWs being suspected of having COVID-19 infection who reported to the fever clinic for the diagnosis of possible COVID-19 by Truenat testing. This included all symptomatic or asymptomatic HCWs and also any HCW who had been declared positive for COVID-19 in the recent past (by Truenat/or any other alternative method) and was coming for a repeat testing. There were no exclusion criteria. The exact sample size could not be calculated since this was a pilot study. All the retrospective data with the effect from the 7th July 2020 were included (when testing was initiated at this hospital) and the study prospectively continued till 15th August when the number was adequate for a timely, meaningful, and relevant conclusion.
The participants were enrolled through a self-reporting COVID-19 Risk Assessment form by Google App. Informed consent was obtained from the HCWs for their inclusion as well as the elaboration of their clinical, social, and demographic details. The Risk Assessment Questionnaire, also containing other relevant information related to the clinic, social or demographic profile of the patient, was used to collect, analyze and interpret the data. These included age, sex, blood group, body mass index (BMI), eating habit whether vegetarian or non-vegetarian, lifestyle in terms of physical activity and average hours of sleep, menstrual or pregnancy history in females, whether symptomatic or asymptomatic at the time of testing, whether on any medication, history of BCG, any previous history of medical illness or allergies or any genetic disorder, job profile and area of posting of the HCW, whether personal protective equipment (PPE) usage was adequate and proper or not at the time of exposure, the type of exposure history, any contact history and whether the HCW had undergone any training on infection prevention and control for COVID-19.

\subsection{Sample Collection, Processing and Discard}

Oropharyngeal swab specimen was collected as per standard procedures using a standard nylon flocked swab. The swab with specimen was inserted into the Viral Transport Medium. Biosafety cabinet II would be used for handling all the specimens. All the biomedical waste items (including the samples) generated from the usage of the kit, after disinfection in 5\% sodium hypochlorite, were disposed of in red bags as per Bio-Medical Waste Management Rules 2016, which were amended in 2018 and 2019.

\subsection{Truenat Principle and Protocol}

Truenat works on the principle of real-time reverse transcription polymerase chain reaction (RT-PCR) based on Taqman chemistry. Truenat ${ }^{\mathrm{TM}}$ Beta $\mathrm{CoV}$ is a chipbased RT-PCR first-line screening test for COVID-19. Truenat ${ }^{\mathrm{TM}}$ SARS CoV-2 is a chip-based RT-PCR to follow on confirmatory test for COVID-19 on Truenat ${ }^{\mathrm{TM}}$ Beta CoV positive samples.

The RNA from the patient sample was first extracted using Trueprep AUTO Universal Cartridge based sample preparation device and Trueprep AUTO Universal Cartridge based sample PrepKit. The Truenat Beta CoV chip was placed on the chip tray of the Truelab ${ }^{\text {tw }}$ Real Time micro $6 \mu \mathrm{L}$ of purified RNA was dispensed into the PCR reagents and allowed to stand for 30 to 60 seconds for a clear solution. Six microliters of this was dispensed into the reaction well of the Truenat Beta CoV chip and the test was inserted into the Truelab ${ }^{\mathrm{TM}}$ real time quantitative micro PCR Analyzer. A positive amplification is indicated by releasing the fluorophores in an exponential manner which is displayed as an amplification curve on the screen. The cycle threshold $(\mathrm{Ct})$ is the number of amplification cycles required for the signal to cross the fluorescence 
threshold, which is inversely proportional to the amount of target nucleic acid in the sample.

\subsection{Statistical Analysis}

The clinical, social, and demographic parameters were compared between those positive by Truenat and those negative by Truenat through chi-square test. Continuous normal variables were represented as means and standard deviation, while non-normal variables were represented as median and interquartile ranges. Comparison of continuous variables between the Truenat positive and Truenat negative was made by unpaired t-test/MannWhitney test.

\section{Results}

Between 7th July and 15th August, 100 HCWs who had got themselves tested for suspected COVID-19 by Truenat, consented to be enrolled in the study. Out of a total of 329 patients who had reported to the fever clinic and were opted for Truenat testing for suspected COVID-19 during the study duration, 100 were front-line HCWs (an additional 5 HCWs did not either consent to get enrolled or could not be contacted); giving an approximate burden of $30.4 \%$ of HCWs reporting for suspected COVID-19 infection.

The mean age of the study group was 29.93 years. The male to female ratio was 0.96 . The age and gender distribution of the study group is detailed in Figure 1. Sixty percent of our HCWs comprised of doctors followed by nurses, nursing officers, technicians and other paramedical staff. Figure 2 shows the distribution of various HCWs among Truenat diagnosed positive and negative subgroups and the frequency of areas of the hospital where these HCWs were posted or got exposed. Sixteen percent of these were posted in the ICU followed by the gynaecology department, fever clinic, COVID ward, and casualty among other commonly posted areas.

Regarding the baseline lifestyle parameters, the majority (84\%) reported 5-8 average hours of sleep. Nine percent reported more than 8 hours, while $7 \%$ slept for less than 5 hours. While $51 \%$ reported being non-vegetarian, $47 \%$ were

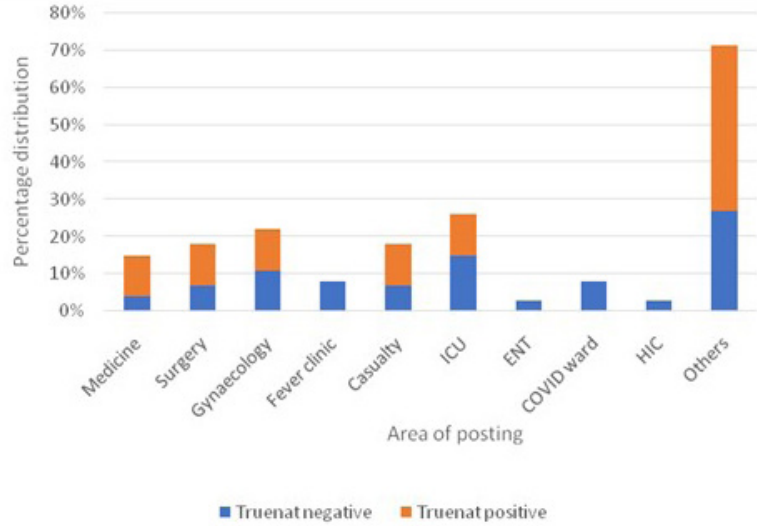

Figure 2. Area of Posting/Work Among Truenat Diagnosed Positive \& Negative Health Care Workers.

vegetarian and the rest were vegans. When asked to selfassess themselves in terms of physical activity, $51 \%$ rated themselves $3 / 5$, while $31 \%, 10 \%$, and $8 \%$ rated themselves $4 / 5,5 / 5$, and $2 / 5$, respectively. The BMI ranged from $18.5-$ 24.9 in $60 \%$ cases while $39 \%$ and $1 \%$ cases had their BMI in the range of 25-29.9 and 30-34.9, respectively. The comparison of these baseline socio-clinical and lifestyle parameters between the Truenat diagnosed positive and negative HCWs is depicted in Table 1. Comparison of mean age and median physical activity (self-graded by study participants) is shown in Table 2.

Table 1 also shows the BCG immunization, blood group and hydroxychloroquine intake among Truenat diagnosed positive and negative HCWs. Among 100 HCWs, 83\% reported either the presence of BCG scar or gave a history of BCG immunization at birth. $\mathrm{O}^{+}$was the most common blood group reported in $38 \%$ of these $\mathrm{HCW}$, followed by $\mathrm{B}^{+}, \mathrm{A}^{+}, \mathrm{AB}^{+}$, and $\mathrm{O}^{-}$in $28 \%, 21 \%, 9 \%$, and $4 \%$ cases, respectively. Seventy-six percent of the HCWs denied taking any prophylactic dose of HCQS among whom $57.89 \%$ were doctors. Among those who took the prophylactic dose, the maximum number of HCWs (29.16\%) took it for four weeks. Table 3 shows the comparison of the mean duration
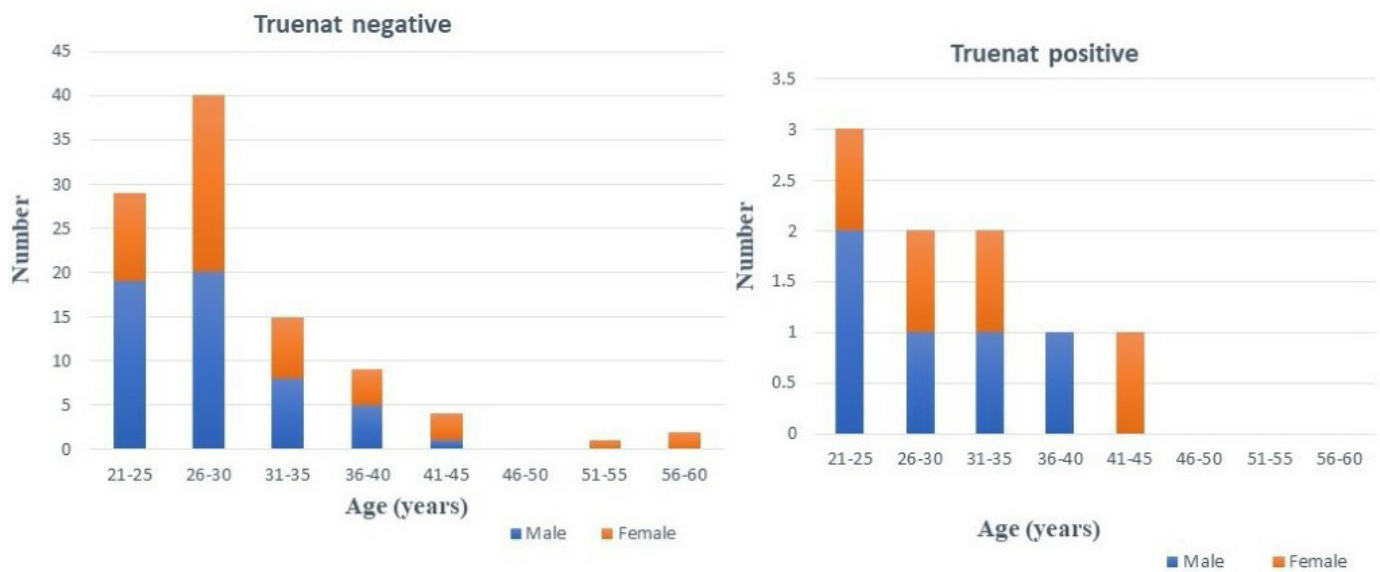

Figure 1 . Age and Gender Distribution Among Truenat Diagnosed Positive \& Negative Health Care Workers. 
Table 1. Baseline Socio-Clinical and Lifestyle Modification Factors Among Truenat Diagnosed Positive \& Negative Health Care Workers

\begin{tabular}{|c|c|c|c|c|c|}
\hline Baseline Parameters & & Total $(n=100)$ & Truenat Positive $(n=9)$ & Truenat Negative $(n=91)$ & $P$ Value \\
\hline \multirow{2}{*}{ Sex } & Male & $52(\% 52)$ & $5(\% 55.5)$ & $47(\% 51.6)$ & \multirow{2}{*}{0.822} \\
\hline & Female & $48(\% 48)$ & $4(\% 44.5)$ & $44(\% 48.4)$ & \\
\hline \multirow{3}{*}{ Type of HCW } & Doctor & $60(\% 60)$ & $3(\% 33.3)$ & $57(\% 62.6)$ & \multirow{3}{*}{0.021} \\
\hline & Nurse/nursing officer & $31(\% 31)$ & $3(\% 33.3)$ & $28(\% 30.8)$ & \\
\hline & Technician/others & $9(\% 9)$ & $3(\% 33.3)$ & $6(\% 6.6)$ & \\
\hline \multirow{2}{*}{$\mathrm{BMI}\left(\mathrm{kg} / \mathrm{m}^{2}\right)$} & Less than 25 & $60(\% 60)$ & $4(\% 44.45)$ & $56(\% 61.5)$ & \multirow{2}{*}{0.298} \\
\hline & 25 and above & $40(\% 40)$ & $5(\% 55.5)$ & $35(\% 38.5)$ & \\
\hline \multirow{3}{*}{ Hours of sleep (hours) } & $<5$ & $7(\% 7)$ & $1(\% 11.1)$ & $6(\% 6.6)$ & \multirow{3}{*}{0.846} \\
\hline & $8-5$ & $84(\% 84)$ & $7(\% 77.7)$ & $77(\% 84.6)$ & \\
\hline & $>8$ & $9(\% 9)$ & $1(\% 11.1)$ & $8(\% 8.8)$ & \\
\hline \multirow{2}{*}{ Diet } & Vegetarian/vegan & $48(\% 48)$ & $5(\% 55.5)$ & $43(\% 46.1)$ & \multirow{2}{*}{0.634} \\
\hline & Non-vegetarian/others & $52(\% 52)$ & $4(\% 44.4)$ & $48(\% 51.6)$ & \\
\hline \multirow{2}{*}{ BCG immunization (History/scar) } & Present & $83(\% 83)$ & $7(\% 77.7)$ & $76(\% 83.5)$ & \multirow{2}{*}{0.662} \\
\hline & Absent & $17(\% 17)$ & $2(\% 22.2)$ & $15(\% 16.48)$ & \\
\hline \multirow{4}{*}{ Blood Group } & A & 21 & 5 & 16 & \multirow{4}{*}{0.072} \\
\hline & B & 28 & 2 & 26 & \\
\hline & $A B$ & 9 & 0 & 9 & \\
\hline & $\mathrm{O}$ & 38 & 2 & 36 & \\
\hline \multirow{2}{*}{ Rh Factor } & + & 96 & 9 & 87 & \multirow{2}{*}{0.521} \\
\hline & - & 4 & 0 & 4 & \\
\hline \multirow{2}{*}{$\begin{array}{l}\text { Prophylactic dose of } \\
\text { Hydroxychloroquine }\end{array}$} & Taken & $27(\% 27)$ & $6(\% 66.6)$ & $24(\% 26.37)$ & \multirow{2}{*}{0.654} \\
\hline & Not taken & $73(\% 73)$ & $6(\% 66.6)$ & $67(\% 73.6)$ & \\
\hline
\end{tabular}

of HCQ prophylaxis between those Truenat positive and negative participants and also between doctors and nondoctor (nurses, technicians) personnel.

While $74 \%$ of the HCWs affirmed the use of PPE at the time of exposure, $18 \%$ denied the use of PPE at the time of exposure. None of them reported a breach in PPE, or inadequate use of PPE. Eight percent of the HCWs were not sure about their usage of PPE at the time of exposure. Among the HCWs who reported to have used PPE at the time of exposure; $60.81 \%$ were doctors, whereas only $16.22 \%-17.57 \%$ of nurses or nursing officers reported the same. On the other hand, among those who reported not to have used PPE at the time of exposure, 55.55\% were doctors and the same was reported only in approximately $22.22 \%$ of the nurses. Whereas $62.5 \%$ of the doctors and $12.5 \%$ of nurses, nursing officers, or other categories of HCWs were not certain about their PPE usage at the time of exposure.

As far as a training programme for the infection control and prevention for COVID-19 is concerned, half of the study population (50\%) reported to have attended such a training programme at some point in time; however, $44 \%$ denied having attended any such training programme. Six percent again were not sure if they had attended any such programme.

The comorbidities reported were hypothyroidism with hypertension and rheumatoid arthritis; chronic sinusitis; hypertension; hypothyroidism with tubercular lymphadenitis; pericarditis; atrial tachycardia; allergic rhinitis; and hypothyroidism. Regarding any menstrual or obstetric abnormality; hypertension in pregnancy, polymenorrhea, dysfunctional uterine bleeding, and bad obstetric history were recorded in $1.96 \%$ (one case each) cases among the 51 females in our study group.

The kind of exposure accounting for the suspected infection that was reported by the HCWs varied. The most common mode reported was direct contact with or exposure to COVID-19 patients in $75 \%$ of cases, either during the rounds taken of such wards or during the care of such patients. The other routes presumptively reported were exposure to colleague HCWs by $6 \%$, exposure to family members by $5 \%$ and exposure while doing an aerosol procedure or while handling samples in the lab by $2 \%$ cases each. Taking rounds in the red containment area of the hospital and exposure to a public place were reported in 1\% cases. Another 1\% reported an indirect exposure, while $7 \%$ were not sure about the source of their suspected infection.

Among the HCWs under study, 74\% were asymptomatic at the time of testing (Figure 3). Figure 4 shows the clinical presentation among Truenat diagnosed positive or negative HCWs. Fever was the most common reported symptom (27\%), while sore throat, dry cough, body pain, general malaise were other common symptoms reported in $18 \%, 16 \%, 11 \%$, and $9 \%$ cases respectively. Rhinorrhoea, anosmia and diarrhea, each were reported in $5 \%$ of cases, 
Table 2. Comparison of Age and Physical Activity Self-grading Between Those Testing Truenat Positive and Truenat Negative

\begin{tabular}{lccc}
\hline & $\begin{array}{c}\text { Truenat Positive } \\
(\mathbf{n}=\mathbf{9})\end{array}$ & $\begin{array}{c}\text { Truenat Negative } \\
(\mathbf{n}=\mathbf{9 1})\end{array}$ & P Value \\
\hline Age, Mean (SD) & $30.78(7.10)$ & $29.85(7.24)$ & 0.713 \\
$\begin{array}{l}\text { Physical activity } \\
\text { grade, Median (IQR) }\end{array}$ & $4(5-3)$ & $3(4-3)$ & 0.198 \\
\hline
\end{tabular}

$\mathrm{IQR}$, interquartile range.

Table 3. Comparison of Mean Duration of HCQ Prophylaxis

\begin{tabular}{lccc}
\hline & \multicolumn{2}{c}{ Mean (SD) } & P Value \\
\cline { 2 - 3 } & $\begin{array}{c}\text { Truenat Positive } \\
(\mathbf{n}=\mathbf{9})\end{array}$ & $\begin{array}{c}\text { Truenat Negative } \\
(\mathbf{n}=\mathbf{9 1})\end{array}$ & \\
\hline $\begin{array}{l}\text { Duration of HCQ } \\
\text { Prophylaxis (wk) }\end{array}$ & $1.00(2.34)$ & $1.19(2.37)$ & 0.822 \\
\hline & $\begin{array}{c}\text { Doctor } \\
(\mathbf{n}=\mathbf{6 0})\end{array}$ & $\begin{array}{c}\text { Non-Doctor } \\
(\mathbf{n}=\mathbf{4 0})\end{array}$ & \\
\hline $\begin{array}{l}\text { Duration of HCQ } \\
\text { Prophylaxis }(\mathrm{wk})\end{array}$ & $1.25(2.35)$ & $1.05(2.40)$ & 0.680 \\
\hline
\end{tabular}

while headache and difficulty in breathing were reported in $2 \%$ of cases each of all the HCWs.

Truenat was reported positive in 9 of the $100 \mathrm{HCW}$ sho got tested, giving an infection rate of $9 \%$. Out of these 100 HCWs, 21 also had got tested by RT-PCR of whom four were positive (19.05\% positivity). Twenty-two of the 100 got themselves tested by rapid antigen testing of whom four reported positive (18.18\% positivity). These tests were reported to be conducted at various stages of their illnesses and not necessarily on the same day as that of Truenat testing. Six of the 100 healthcare workers under study had got their antibodies tested prospectively and four of them $(66.67 \%)$ reported the documentation of antibodies. Out of the six that got tested, only four were positive by Truenat. All these four positives documented the presence of antibodies.

When all these parameters were compared statistically among Truenat diagnosed positive and negative HCWs, only being asymptomatic or symptomatic at the time of Truenat testing was found to be significantly associated with the positivity by Truenat. When they were inquired about their current status of infection, 89\% reported being asymptomatic negative. While the rest were either symptomatic negative $(5 \%)$, symptomatic positive $(4 \%)$ or asymptomatic positive (2\%).

\section{Discussion}

In November 2002, a novel beta coronavirus called SARS coronavirus (SARS-CoV) emerged in Guangdong, China, which led to more than 8000 infections and 774 deaths in 37 countries. In 2012, MERS coronavirus (MERS-CoV), affected 2494 individuals and caused 858 deaths. ${ }^{3,4}$ Since emerging in Wuhan, in December 2019, the COVID-19 epidemic caused by SARS-CoV-2 has progressed rapidly into a pandemic. The initial cases were linked to direct exposure to infected animals showing animal-to-human transmission at a seafood market in China, though, clinical cases with diversity in exposure history have emerged now.
This helps to further elaborate that human-to-human transmission of the virus is also possible, which is now considered the main form of transmission. Transmission generally occurs from the aerosolized droplets through coughing or sneezing, also among asymptomatic individuals. Close contact between individuals can also result in transmission, which also indicates possible transmission in closed spaces due to increased aerosol concentrations. ${ }^{5}$

Data on the risk or magnitude of COVID-19 among front-line HCWs are limited. Studies suggest that frontline HCWs have a significantly increased risk of COVID-19 infection compared to the general population and such infections were the highest among those HCWs who had inadequate access to PPE or who reused their PPE. However, adequate supplies of PPE do not completely mitigate high-risk exposures and infections. This highlights the importance of not only ensuring adequate PPE availability of good quality but also emphasizing the other aspects of proper usage of PPE, like correct donning, doffing, and the rational use in an appropriate clinical situation. ${ }^{6-8}$

The median age reported was 18-40 years. An Indian study conducted on the prevalence of COVID-19 among HCWs, reports almost concordant findings. ${ }^{9}$ Male to female ratio of 2.03, unlike 0.93 in our study, was reported among the HCWs in a study on the screening of asymptomatic HCWs who took care of patients infected with novel corona

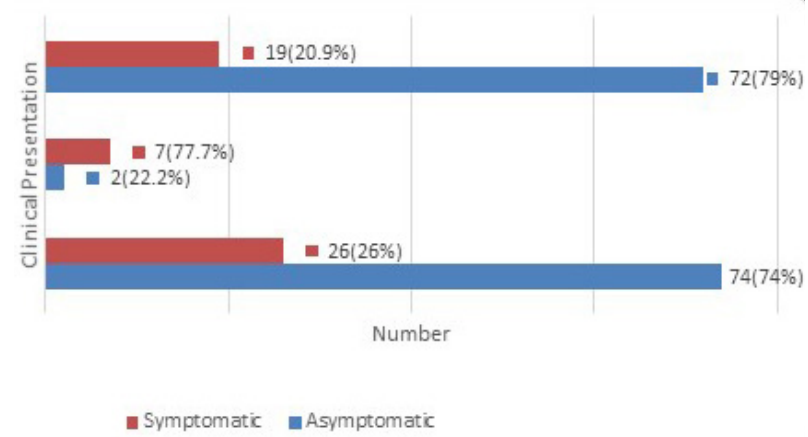

Figure 3. Symptomatic and Asymptomatic Presentation Among Truenat Diagnosed Positive \& Negative Health Care Workers. $P$ value $=0.001, \chi^{2}=13.78$.

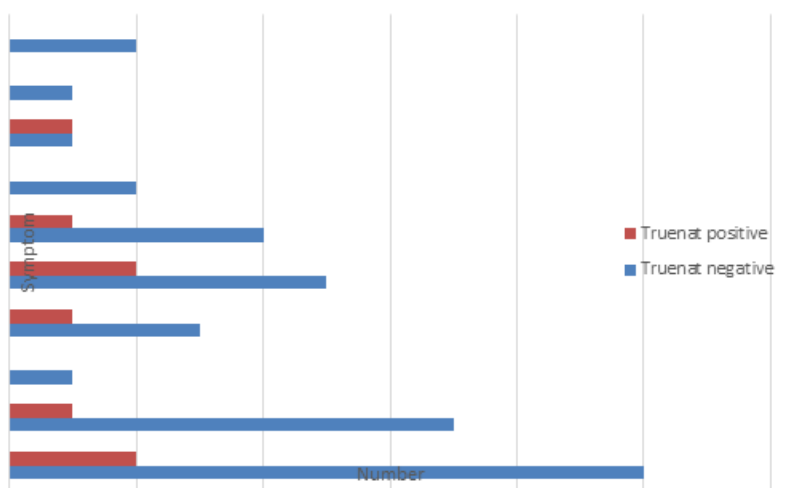

Figure 4. Clinical Presentation Among Truenat Diagnosed Positive \& Negative Health Care Workers 
virus. ${ }^{10}$ The same study reported a prevalence of positiveCOVID-19 as 0\% among asymptomatic HCWs, though the researchers found the result unexpected considering the high-risk profile of HCWs. Some other possible explanations that the researchers have hypothesized were an asymptomatic presentation of the majority of their patients, false negativity of RTPCR among asymptomatic cases with low viral load, or the use of nasopharyngeal swabs. ${ }^{10}$ They further stated that extreme prevention measures could also result in such a low prevalence. In our study, however, $2.7 \%$ of the asymptomatic HCWs were positive by Truenat, whereas $73.1 \%$ of the symptomatic HCWs were reported negative. However, similar to our study, they too found the symptomatic status to be the single statistically significant and important predictor of infection. The same study has notably reported a $0.52 \%$ infection rate among nurses as unlike $11 \%$ in our case which still was much lower than $34 \%$ among the doctors. ${ }^{10}$ This is in contrast with the findings of a KAP survey done among HCWs for COVID-19, according to which, doctors showed a higher knowledge score than nurses and also found that knowledge directly affected the attitude of the HCW towards work. The authors have suggested that preventive training should be organised considering factors like experience, education, etc. as knowledge is a prerequisite for the establishment of positive attitude and firm beliefs. ${ }^{11}$

Rapid and accurate detection of COVID-19 is pivotal in controlling outbreaks in the community and hospitals. Current diagnostic tests for COVID-19 include RT-PCR. Though nucleic acid tests are rapid and sensitive diagnostic tools for COVID-19 patients, genetic variation and mismatches of primers, probes, and target sequences may result in reduced detection performance and false-negative results. Suspects with positive chest computed tomography scans can get negative results by RT-PCR. Furthermore, specimens with low viral RNA load may be identified as negative. Therefore, a careful interpretation of nucleic acid test results must be concluded. Serological surveys can contribute to the investigation of an ongoing outbreak and retrospective assessment of the attack rate or extent of an outbreak. ${ }^{6}$ With the surge in cases of COVID-19 in India and over 150 countries across the globe, a rapid point of care assay is considered to be a major goal towards the containment of cases. Truenat is a chip-based RT-PCR test for the semi-quantitative detection of Beta Coronavirus and SARS Coronavirus RNA. The target sequence is the E gene of Sarbeco virus for beta Coronavirus and Rdrp gene for SARS Coronavirus. The Real Time Micro PCR system is achieved through a combination of the cartridge-based RNA extraction system and Real Time Micro PCR analyzer. Testing of single sample initiating from RNA extraction till getting real-time results takes only about 1 hour. Minimal training is required to do the procedure and there is no need to prepare the master mix. The test equipment is light and portable and hence ideal for screening in field settings with the added benefit of a short turnaround time.
One point eight percent prevalence was reported in a cohort of HCWs from a similar geographical area, though the method of testing was not specified. ${ }^{9}$ They similar to the results of our study found no statistical correlation between factors such as an area of posting or PPE usage and positivity for COVID-19. The authors also reported higher positivity (5.2\%) among symptomatic HCWs as compared to those who were asymptomatic (1.1\%), similar to the result in our study (26.9 vs $2.7 \%$ ). Another study performed in Italy found a COVID-19 prevalence of $3.4 \%$ among HCWs. ${ }^{12}$ Comparatively higher rate in our study could be possibly because of the fact that ours was not a screening programme meant for only asymptomatic HCWs. The intrafamilial transmission has been pointed out in this Italian study which was also witnessed in $5 \%$ of cases of our study. ${ }^{12}$ However the proportion of HCWs having undergone training in their study is much higher (almost 100\%) than what was witnessed in our study (50\%). Despite implementation of a mandatory Institutional Training Programme, such a low figure definitely points out the need for a much greater emphasis on such awareness programmes.

HCQS was taken only by $24 \%$ of our HCWs, which is quite low as compared to the study which reports HCQS intake by $67.8 \%$ HCW s. ${ }^{9}$ The same study reported a positivity of $1.9 \%$ and $1.7 \%$ among those who took a prophylactic dose when compared to those who did not, though this was not statistically significant similar to our finding. In our study, $8.3 \%$ of those who took the prophylactic dose tested positive as compared to $9.21 \%$ positives among those who did not take prophylactic HCQS.

\section{Conclusion}

Though a rapid but vigorous response by the global scientific community has described many crucial aspects of SARS-CoV-2 transmission and its natural history, key questions remain. ${ }^{7}$ If well-tracked, early introduction of an emerging pathogen provides a unique opportunity to characterize its transmission, natural history, the unseen burden of infection, and the effectiveness of screening. ${ }^{8}$

\section{Authors' Contributions}

BK, RJ, NPS, KS, RA and AK made substantial contributions to the conception and design, to the acquisition, analysis, and interpretation of the data; $\mathrm{BK}, \mathrm{RJ}$ and $\mathrm{KS}$ contributed to the drafting of the article or critical revision for important intellectual content for the final approval of the version to be published by BK, RJ, NPS, KS, RA \& AK. BK, RJ, NPS, $\mathrm{KS}, \mathrm{RA}$ and AK agree to be accountable for all aspects of the work ensuring that questions related to the accuracy or integrity of any part of the article are appropriately investigated and resolved.

\section{Conflict of Interest Disclosures}

The authors declare that they have no conflicts of interest. 
Research Highlights

\section{What Is Already Known?}

- In December 2019, in the city of Wuhan, China; a new coronavirus emerged, that had not been previously identified in humans

- HCWs are exposed to hazards that put them at risk of infection with an outbreak pathogen as they are at the front line of any outbreak response.

\section{What Does This Study Add?}

- Truenat was reported positive in 9 of the $100 \mathrm{HCWs}$ who got tested, giving an infection rate of $9 \%$.

- The symptomatic status is found to be the single statistically significant and important predictor of infection.

\section{Ethical Approval}

Ethical approval was obtained from the Institutional Ethics Committee-Human research of the University College of Medical Sciences with code of IEC-HR/2020/44/13R2.

\section{References}

1. Lu R, Zhao X, Li J, et al. Genomic characterisation and epidemiology of 2019 novel coronavirus: implications for virus origins and receptor binding. Lancet. 2020;395(10224):565574. doi:10.1016/s0140-6736(20)30251-8.

2. de Wit E, van Doremalen N, Falzarano D, Munster VJ. SARS and MERS: recent insights into emerging coronaviruses. Nat Rev Microbiol. 2016;14(8):523-534. doi:10.1038/ nrmicro.2016.81.

3. Li Q, Guan X, Wu P, et al. Early transmission dynamics in Wuhan, China, of novel coronavirus-infected pneumonia.
N Engl J Med. 2020;382(13):1199-1207. doi:10.1056/ NEJMoa2001316.

4. Ge H, Wang X, Yuan X, et al. The epidemiology and clinical information about COVID-19. Eur J Clin Microbiol Infect Dis. 2020;39(6):1011-1019. doi:10.1007/s10096-020-03874-z.

5. Nguyen LH, Drew DA, Graham MS, et al. Risk of COVID-19 among front-line health-care workers and the general community: a prospective cohort study. Lancet Public Health. 2020;5(9):e475-e483. doi:10.1016/s2468-2667(20)30164-x.

6. Zheng L, Wang X, Zhou C, et al. Analysis of the infection status of healthcare workers in Wuhan during the COVID-19 outbreak: a cross-sectional study. Clin Infect Dis. 2020;71(16):21092113. doi:10.1093/cid/ciaa588.

7. Jha S, Soni A, Siddiqui S, et al. Prevalence of flu-like symptoms and COVID-19 in healthcare workers from India. J Assoc Physicians India. 2020;68(7):27-29.

8. Al-Zoubi NA, Obeidat BR, Al-Ghazo MA, et al. Prevalence of positive COVID-19 among asymptomatic health care workers who care patients infected with the novel coronavirus: a retrospective study. Ann Med Surg (Lond). 2020;57:14-16. doi:10.1016/j.amsu.2020.06.038.

9. Ellison RT III. COVID-19 in Healthcare Workers. https:// www.jwatch.org/na51848/2020/07/01/covid-19-healthcareworkers. Published July 1, 2020.

10. Lai X, Wang M, Qin C, et al. Coronavirus disease 2019 (COVID-2019) infection among health care workers and implications for prevention measures in a tertiary hospital in Wuhan, China. JAMA Netw Open. 2020;3(5):e209666. doi:10.1001/jamanetworkopen.2020.9666.

11. Zhang M, Zhou M, Tang F, et al. Knowledge, attitude, and practice regarding COVID-19 among healthcare workers in Henan, China. J Hosp Infect. 2020;105(2):183-187. doi:10.1016/j.jhin.2020.04.012.

12. Sahu AK, Amrithanand VT, Mathew R, Aggarwal P, Nayer J, Bhoi S. COVID-19 in health care workers - a systematic review and meta-analysis. Am J Emerg Med. 2020;38(9):1727-1731. doi:10.1016/j.ajem.2020.05.113. 